\title{
Block-exoticity of a family of exotic fusion systems
}

\author{
Patrick Serwene \\ Communicated by Christopher W. Parker
}

\begin{abstract}
We prove that each exotic fusion system $\mathcal{F}$ on a Sylow $p$-subgroup of $G_{2}(p)$ for an odd prime $p$ with $\mathcal{O}_{p}(\mathcal{F})=1$ is block-exotic. This gives evidence for the conjecture that each exotic fusion system is block-exotic. We prove two reduction theorems for blockrealisable fusion systems.
\end{abstract}

\section{Introduction}

Let $p$ be a prime number and $k$ an algebraically closed field of characteristic $p$. Let $P$ a finite $p$-group and $\mathscr{F}$ a fusion system on $P$. By fusion system, we always mean saturated fusion system. Recall that $\mathscr{F}$ is said to be realisable if $\mathscr{F}=\mathscr{F}_{P}(G)$ for a finite group $G$ and $P \in \operatorname{Syl}_{p}(G)$; otherwise $\mathcal{F}$ is said to be exotic. Also recall that if $\mathcal{F}=\mathscr{F}_{\left(P, e_{P}\right)}(G, b)$ for a finite group $G$ having a $p$-block $b$ with maximal $b$-Brauer pair $\left(P, e_{P}\right), \mathcal{F}$ is said to be block-realisable; otherwise $\mathcal{F}$ is said to be block-exotic. By a $p$-block, we mean a primitive central idempotent of $k G$. See Section 2 for details.

The following fact is a consequence of Brauer's third main theorem (see [7, Theorem 7.1]): If $G$ is a finite group and $b$ is the principal $p$-block of $k G$, i.e. the block corresponding to the trivial character, with maximal $b$-Brauer pair $\left(P, e_{P}\right)$, then $P \in \operatorname{Syl}_{p}(G)$ and $\mathcal{F}_{\left(P, e_{P}\right)}(G, b)=\mathcal{F}_{P}(G)$. In particular, any realisable fusion system is block-realisable. The converse is still an open problem. However, we have the following conjecture.

Conjecture 1.1. If $\mathcal{F}$ is an exotic fusion system, then $\mathscr{F}$ is block-exotic.

There have been only two families of exotic fusion systems for which blockexoticity has been proven. The first one being the Solomon systems defined on a Sylow 2-subgroup of $\operatorname{Spin}_{7}(q)$. These are conjectured to be the only exotic fusion systems on 2-groups. The block-exoticity for $q=3$ was proven in [7] and generalised to all odd prime powers $q$ in [3, Theorem 9.34]. The second example consists of the Ruiz-Viruel systems, which are defined on the extra special 7-group of order $7^{3}$ and for which block-exoticity has been proven in [9]. 
In this paper, we provide further evidence for Conjecture 1.1. Our first main result is the following.

Theorem 1.2. Conjecture 1.1 is true for all fusion systems $\mathcal{F}$ on a Sylow $p$-subgroup of $G_{2}(p)$ for odd $p$ with $\mathcal{O}_{p}(\mathcal{F})=1$.

The fusion systems on a Sylow $p$-subgroup of $G_{2}(p)$ for odd $p$ and $\mathcal{O}_{p}(\mathcal{F})=1$ have been classified by Parker and Semeraro in [12], and thus we refer to them as Parker-Semeraro systems. For $p \neq 7$, all Parker-Semeraro systems are realised by finite groups, whereas for $p=7$, there are 29 Parker-Semeraro systems of which 27 are exotic. In this paper, we prove block-exoticity of these.

The relevance of the Parker-Semeraro systems stems from the fact that one wants to classify all fusion systems over maximal unipotent subgroups of finite groups of Lie type of small rank. Note that the Solomon systems belong to this class of fusion systems as well. Furthermore, another important factor is that 7 is a good prime, and thus many results for groups of Lie type will be applicable.

The proof of Theorem 1.2 uses the classification of finite simple groups. A key step is a reduction theorem which we state after the following definition.

Definition 1.3. Let $\mathscr{F}$ be a fusion system on a finite $p$-group $P$. If $P$ has no nontrivial proper strongly $\mathcal{F}$-closed subgroups, we call $\mathcal{F}$ reduction simple.

Note that if $\mathcal{F}=\mathscr{F}_{\left(P, e_{P}\right)}(G, b)$, then $b$ is said to be an $\mathcal{F}_{\text {-block. Recall the }}$ following normal subgroups of a finite group, which will be important in one of our reduction theorems.

Definition 1.4. Let $G$ be a finite group.

(a) The unique maximal semisimple normal subgroup of $G$ is called the layer of $G$ and denoted by $E(G)$. If $E(G) \neq 1$, the uniquely determined quasisimple factors of $G$ are called the components of $G$.

(b) The unique maximal nilpotent normal subgroup of $G$ is called the Fitting subgroup of $G$ and denoted by $F(G)$.

The reduction we apply to prove Theorem 1.2 takes the following form.

Theorem 1.5. Let $\mathcal{F}$ be a reduction simple fusion system on a non-abelian $p$ group $P$. If $\mathcal{F}$ is block-realisable, then there exists a finite group $G$ possessing an $\mathcal{F}$-block $b$ such that the following holds.

(a) $|G: Z(G)|$ is minimal among all groups $G$ having an $\mathcal{F}$-block. 
(b) If $H \unlhd G$ with $P \nsubseteq H$, then $H$ is a central $p^{\prime}$-group. In particular, we have $F(G)=Z(G)$.

(c) The number of components of $G$ is bounded by the rank of $Z(P)$.

Our final main result is another reduction theorem, which generalises [9, Theorem 4.2]. We refer to Section 2 for terminology.

Theorem 1.6. Let $\mathcal{F}$ be a fusion system on a non-abelian p-group P. Denote by

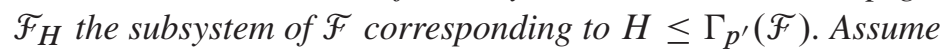

(a) $\mathcal{O}^{p^{\prime}}(\mathcal{F})$ is reduction simple,

(b) if $\mathcal{E}$ is a fusion system on $P$ containing $\mathcal{O}^{p^{\prime}}(\mathcal{F})$, then $\mathscr{G} \subseteq \mathcal{F}$,

(c) if $\mathscr{E}$ is a fusion system on $P$ such that $\mathscr{E} \unlhd \mathscr{F}_{H}$, then $\mathcal{O}^{p^{\prime}}(\mathscr{F}) \subseteq \mathscr{E}$.

If there exists a finite group having an $\mathcal{F}_{H}$-block, then there exists a finite quasisimple group $L$ with $p^{\prime}$-centre having an $\widetilde{F}_{H^{\prime}}$-block for some $H^{\prime} \leq \Gamma_{p^{\prime}}(\mathcal{F})$.

In the next section, we recall the definition of fusion systems, several of their key properties and results. Section 3 will be concerned with reduction results, and we prove Theorems 1.5 and 1.6. Finally, Section 4 will be about the Parker-Semeraro systems, and we prove Theorem 1.2.

\section{Background and quoted results on fusion systems}

We begin by recalling the definition of a fusion system.

Definition 2.1. Let $p$ be a prime and $P$ a finite $p$-group. A saturated fusion system on $P$ is a category $\mathscr{F}$ such that $\mathrm{Ob}(\mathcal{F})$ is the set of all subgroups of $P$, and furthermore, for all $Q, R \leq P$, we have

(i) $\operatorname{Hom}_{P}(Q, R) \subseteq \operatorname{Hom}_{\mathcal{F}}(Q, R) \subseteq \operatorname{Inj}(Q, R)$,

(ii) each homomorphism in $\mathcal{F}$ is the composition of an $\mathcal{F}$-isomorphism and an inclusion,

(iii) each subgroup of $P$ is $\mathscr{F}$-conjugate to a subgroup which is fully automised and receptive in $\mathcal{F}$.

We need to recall some of the notation from this definition.

Definition 2.2. Let $\mathcal{F}$ be a fusion system on a $p$-group $P$.

(a) Two subgroups $Q, R \leq P$ are called $\mathcal{F}$-conjugate if they are isomorphic as objects of the category $\mathcal{F}$. 
(b) A subgroup $Q \leq P$ is called fully automised in $\mathcal{F}$ if

$$
\operatorname{Aut}_{P}(Q) \in \operatorname{Syl}_{p}\left(\operatorname{Aut}_{\mathcal{F}}(Q)\right) .
$$

(c) A subgroup $Q \leq P$ is called receptive in $\mathscr{F}$ if, for each $R \leq P$ and each $\varphi \in \operatorname{Iso}_{\mathcal{F}}(R, Q), \varphi$ has an extension to the group

$$
N_{\varphi}:=\left\{g \in N_{P}(R) \mid{ }^{\varphi} c_{g} \in \operatorname{Aut}_{P}(Q)\right\} .
$$

For convenience, we drop the term saturated, and mean saturated fusion system whenever we say fusion system in this paper. In the literature, fusion system means categories satisfying only axioms (i) and (ii) from Definition 2.1. Furthermore, note that a category whose objects consist of the subgroups of some $p$-group $P$ is called a category on $P$.

Theorem 2.3. Let $G$ be a finite group with $P \in \operatorname{Syl}_{p}(G)$. We denote the category on $P$ with morphisms consisting of conjugation by elements in $G$ by $\mathscr{F}_{P}(G)$. Then $\widetilde{F}_{P}(G)$ is a fusion system on $P$.

If a fusion system is of the form defined in the previous theorem, we call it realisable, otherwise we call it exotic.

Definition 2.4. Let $\mathcal{F}$ be a fusion system on a $p$-group $P$ and $\mathcal{E} \subseteq \mathcal{F}$ a subcategory of $\mathscr{F}$ which is a fusion system itself on some subgroup $P^{\prime} \leq P$.

(a) A subgroup $Q \leq P$ is called strongly $\mathcal{F}$-closed, if

$$
\varphi(R) \subseteq Q \quad \text { for each } \varphi \in \operatorname{Hom}_{\mathscr{F}}(R, P) \text { and each } R \leq Q .
$$

(b) If $P^{\prime}$ is normal in $P$ and strongly $\mathcal{F}$-closed, ${ }^{\alpha} \mathcal{E}=\mathscr{E}$ for each $\alpha \in \operatorname{Aut}_{\mathscr{F}}\left(P^{\prime}\right)$ and for each $Q \leq P^{\prime}$ and $\varphi \in \operatorname{Hom}_{\mathscr{F}}\left(Q, P^{\prime}\right)$, there are $\alpha \in \operatorname{Aut}_{\mathscr{F}}\left(P^{\prime}\right)$ and $\varphi_{0} \in \operatorname{Hom}_{\mathcal{E}}\left(Q, P^{\prime}\right)$ with $\varphi=\alpha \circ \varphi_{0}$, then $\mathcal{E}$ is called weakly normal in $\mathcal{F}$, denoted $\mathcal{E} \dot{\unlhd} \mathscr{F}$.

(c) If $\mathcal{E}$ is weakly normal and, in addition, we have that each $\alpha \in \operatorname{Aut}_{\mathcal{E}}\left(P^{\prime}\right)$ has an extension $\bar{\alpha} \in \operatorname{Aut}_{\mathscr{F}}\left(P^{\prime} C_{P}\left(P^{\prime}\right)\right)$ with $\left[\bar{\alpha}, C_{P}\left(P^{\prime}\right)\right] \leq Z\left(P^{\prime}\right)$, then we call $\mathcal{E}$ normal in $\mathcal{F}$ and write $\mathcal{E} \unlhd \mathscr{F}$.

(d) A fusion system is called simple if it does not contain any non-trivial proper normal subsystem.

The collection of weakly normal subsystems of $\mathscr{F}$ on $P$, ordered by inclusion, has a unique minimal element; see [1, Theorem 7.7]. We denote this subsystem by $\mathcal{O}^{p^{\prime}}(\mathcal{F})$. Additionally, if $\mathcal{F}$ is a fusion system on $P$ and $M \subseteq \operatorname{Aut}_{\mathcal{F}}(Q)$ for some $Q \leq P$, we denote by $\langle M\rangle$ the smallest (not necessarily saturated) fusion system on $P$ such that its morphisms contain $M$. 
Definition 2.5. Let $\mathcal{F}$ be a fusion system on a finite $p$-group $P$.

(a) A subgroup $Q$ of $P$ is called $\mathcal{F}$-centric if $C_{P}\left(Q^{\prime}\right)=Z\left(Q^{\prime}\right)$ for each $Q^{\prime} \leq P$ which is $\mathcal{F}$-conjugate to $Q$.

(b) We say that a subsystem of $\mathcal{F}$ has index prime to $p$ (or $p^{\prime}$-index) if it contains $\mathcal{O}^{p^{\prime}}(\widetilde{F})$.

(c) We define the groups

$$
\begin{aligned}
& \mathcal{O}_{*}^{p^{\prime}}(\mathcal{F}):=\left\langle O^{p^{\prime}}\left(\operatorname{Aut}_{\mathcal{F}}(Q)\right) \mid Q \leq P\right\rangle, \\
& \operatorname{Aut}_{\mathscr{F}}^{0}(\mathcal{F}):=\left\langle\alpha \in \operatorname{Aut}_{\mathcal{F}}(P)|\alpha|_{Q} \in \operatorname{Hom}_{\mathcal{O}_{*}^{p^{\prime}}(\mathcal{F})}(Q, P)\right. \\
& \quad \text { for some } \mathcal{F}_{\text {-centric } Q \leq P\rangle,} Q \leq \operatorname{Aut}_{\mathscr{F}}^{0}(P) .
\end{aligned}
$$

Note that the above definition makes sense since clearly $\mathcal{O}_{*}^{p^{\prime}}(\mathcal{F}) \leq \mathcal{F}$ and also $\operatorname{Aut}_{\mathcal{F}}^{0}(P) \unlhd \operatorname{Aut}_{\mathcal{F}}(P)$.

It turns out that the group $\Gamma_{p^{\prime}}(\mathcal{F})$ carries a lot of information about $\mathcal{F}$.

Theorem 2.6 ([1, Theorem 7.7]). Let $\mathcal{F}$ be a fusion system on a p-group P. There is a one-to-one-correspondence between the subsystems of $\mathcal{F}$ of index prime to $p$ and subgroups of $\Gamma_{p^{\prime}}(\mathcal{F})$. For some $H \leq \Gamma_{p^{\prime}}(\mathcal{F})$, we will refer to the corresponding subsystem by $\tilde{F}_{H}$. Furthermore, this correspondence respects (weak) normality.

For the fusion systems we encounter in this article, the group $\Gamma_{p^{\prime}}(\mathcal{F})$ will always be cyclic.

Definition 2.7. Let $\mathcal{F}$ be a fusion system on a $p$-group $P$. We call a subgroup $Q \leq P$ normal in $\mathscr{F}$, denoted $Q \unlhd \mathscr{F}$, if $Q \unlhd P$ and any morphism

$$
\varphi \in \operatorname{Hom}_{\mathscr{F}}(R, S) \text { for } R, S \leq P
$$

has an extension $\bar{\varphi} \in \operatorname{Hom}_{\mathscr{F}}(R Q, S Q)$ with $\bar{\varphi}(Q)=Q$. The largest subgroup of $P$ normal in $\mathcal{F}$ is denoted by $\mathcal{O}_{p}(\mathcal{F})$.

Lemma 2.8. Let $\mathcal{F}, \mathcal{E}$ be fusion systems on a finite $p$-group $P$ such that $\mathcal{F} \leq \mathcal{E}$, and let $Q \unlhd P$. If $Q$ is normal in $\mathcal{E}$, then $Q$ is normal in $\mathcal{F}$.

Proof. By [1, Proposition 4.5], this is equivalent to showing that $Q$ is contained in each $\mathcal{F}$-essential subgroup $R$ of $P$, and for each of these $R$, we have that $Q$ is Aut $\mathscr{F}(R)$-invariant. Since $Q$ is normal in $\mathcal{E}$, it is strongly $\mathcal{F}$-closed. In particular, $Q$ is $\operatorname{Aut}_{\mathcal{F}}(R)$-invariant for all $R \leq P$ such that $Q \leq R$. 
Now let $\varphi: R \rightarrow T$ be a $\mathcal{G}$-isomorphism. For the other property, we first claim $N_{P}(R) \cap Q \leq N_{\varphi}^{\mathscr{E}}$. Indeed, since $Q \unlhd \mathscr{G}, \varphi$ extends to $\bar{\varphi}: Q R \rightarrow Q T$. Now, for $n \in N_{P}(R) \cap Q$, if we consider the map $c_{n}: R \rightarrow R$, then

$$
{ }^{\varphi} c_{n}=\varphi \circ c_{n} \circ \varphi^{-1}=c_{\bar{\varphi}(n)} \in \operatorname{Aut}_{P}(T),
$$

which means $n \in N_{\varphi}^{\mathcal{E}}$.

Now let $R \leq P$ be $\mathcal{F}_{\text {-essential and }} \beta \in \operatorname{Aut}_{\mathscr{F}}(R)$ such that $N_{\beta}^{\mathscr{F}}=R$ (such a $\beta$ exists since $R$ is $\mathscr{F}$-essential). One easily verifies $N_{\beta}^{\mathscr{F}}=N_{\beta}^{\mathscr{B}}$. So, by the above, we have $N_{P}(R) \cap Q \leq N_{\beta}^{\mathscr{F}}=R$. Since $Q \unlhd P$, we have $R Q \leq P$. By general properties of $p$-groups, either $R Q=R$ or $R<N_{R Q}(R)$. Since we have $N_{R Q}(R)=R N_{Q}(R)=R\left(N_{P}(R) \cap Q\right)=R$, we deduce $R Q=R$, so $Q \leq R$, which implies the claim.

Lemma 2.9. If $\mathcal{F}, \mathcal{E}$ are fusion systems on a finite $p$-group $P$ with $\mathscr{E} \dot{\unlhd} \mathcal{F}$, then $O_{p}(\mathscr{G})$ is normal in $\mathcal{F}$.

Proof. We have to check that each morphism $\varphi \in \operatorname{Hom}_{\mathcal{F}}(Q, P)$ has an extension $\bar{\varphi} \in \operatorname{Hom}_{\mathscr{F}}\left(Q O_{p}(\mathcal{E}), P\right)$ with $\bar{\varphi}\left(O_{p}(\mathscr{G})\right)=O_{p}(\mathscr{E})$. Since $\mathscr{G} \dot{\unlhd} \mathcal{F}, \varphi$ can be written as $\varphi=\alpha \circ \beta$, where $\alpha \in \operatorname{Aut}_{\mathcal{F}}(P)$ and $\beta \in \operatorname{Hom}_{\mathcal{E}}(Q, P)$. Given that $\beta$ is a morphism in $\mathscr{E}$, it extends to $Q O_{p}(\mathscr{E})$. So we only need to show that $\alpha \in \operatorname{Aut}_{\mathcal{F}}(P)$ preserves $O_{p}(\mathscr{E})$. Now, given that $\mathscr{E}$ is normal in $\mathcal{F}$, an $\mathcal{F}$-automorphism of $P$ sends any $\mathcal{E}$-normal subgroup of $P$ to a $\mathcal{E}$-normal subgroup of $P$. In particular, $\alpha\left(O_{p}(\mathscr{E})\right) \subseteq O_{p}(\mathscr{G})$.

Since fusion systems which do not allow many strongly closed subgroups play an important role, we define the following.

Definition 2.10. Let $\mathcal{F}$ be a fusion system on a $p$-group $P$. If $P$ does not have any non-trivial proper strongly $\mathscr{F}$-closed subgroups, we call $\mathscr{F}$ reduction simple.

The rest of this section will recollect some block-theoretic results. In particular, the next theorem shows how blocks of finite groups provide fusion systems.

Definition 2.11. Let $G$ be a finite group and $b$ a block of $k G$. A Brauer pair is a pair $(Q, f)$ where $Q$ is a $p$-subgroup of $G$ and $f$ is a block of $k C_{G}(Q)$. We denote the set of blocks of $k C_{G}(Q)$ for some $p$-subgroup $Q$ of $G$ by $B(Q)$.

Note that $G$ acts on the set of Brauer pairs by conjugation. We recall the Brauer map to see how Brauer pairs form a poset.

Definition 2.12. Suppose $G$ is a finite group, and let $Q \leq G$. For an element $a=\sum_{g \in G} \alpha_{g} g \in k G$, set $\operatorname{Br}_{Q}(a):=\sum_{g \in C_{G}}(Q) \alpha_{g} g$. 
Definition 2.13. Let $(Q, f)$ and $(R, e)$ be Brauer pairs. Then

(a) $(Q, f) \unlhd(R, e)$ if $Q \unlhd R, f$ is $R$-stable and $\operatorname{Br}_{R}(f) e=e$,

(b) $(Q, f) \leq(R, e)$ if $Q \leq R$ and there exist Brauer pairs $\left(S_{i}, d_{i}\right), 1 \leq i \leq n$, such that $(Q, f) \unlhd\left(S_{1}, d_{1}\right) \unlhd\left(S_{2}, d_{2}\right) \unlhd \cdots \unlhd\left(S_{n}, d_{n}\right) \unlhd(R, e)$.

See [7, Section 2] for details. It is actually the case that, for a given Brauer pair $(R, e)$ and some $Q \leq R$, there exists a unique $f \in \mathcal{B}(Q)$ with $(Q, f) \leq(R, e)$.

Definition 2.14. Let $b$ be a block of a finite group $G$.

(a) A $b$-Brauer pair is a Brauer pair $(R, e)$ such that $(1, b) \leq(R, e)$, or equivalently, it is a Brauer pair $(R, e)$ such that $\operatorname{Br}_{R}(b) e=e$.

(b) We denote the blocks $e$ of $k C_{G}(R)$ such that $(1, b) \leq(R, e)$ by $\mathcal{B}(R, b)$.

(c) A defect group of $b$ is a $p$-subgroup $P$ of $G$ maximal such that $\operatorname{Br}_{P}(b) \neq 0$.

Note that the group $G$ acts by conjugation on the set of $b$-Brauer pairs. Furthermore, some $p$-subgroup $P$ of $G$ is a defect group of $b$ if and only if there is a maximal pair $(P, e)$ such that $(1, b) \leq(P, e)$. We refer to such a pair as a maximal $b$-Brauer pair.

Theorem 2.15 ([7, Theorem 3.9 (i)]). Let $b$ be a block of $k G$ and $\left(P, e_{P}\right)$ a maximal $b$-Brauer pair. For a subgroup $Q \leq P$, denote by $e_{Q}$ the unique block such that $\left(Q, e_{Q}\right) \leq\left(P, e_{P}\right)$. Denote the category on $P$ whose morphisms consist of all injective group homomorphisms $\varphi: Q \rightarrow R$ for which there is some $g \in G$ such that $\varphi(x)={ }^{g} x$ for all $x \in Q$ and ${ }^{g}\left(Q, e_{Q}\right) \leq\left(R, e_{R}\right)$ by $\mathcal{F}_{\left(P, e_{P}\right)}(G, b)$. Then $\mathcal{F}_{\left(P, e_{P}\right)}(G, b)$ is a fusion system on $P$.

If a fusion system is of the form defined in the previous theorem, we call it block-realisable; otherwise we call it block-exotic.

Note that, for several of the reduction theorems, we need to introduce more general structures than block fusion systems since some group theoretic properties are not captured by these: Assume $b$ is a block of $k G$ with maximal $b$-Brauer pair $(P, e)$ and $N \unlhd G$. If $c$ is a block of $k N$ covered by $b$, i.e. $b c \neq 0, P \cap N$ will be a defect group for $c$. However, in general, $\mathcal{F}_{\left(P \cap N, e_{P \cap N}\right)}(N, c)$ does not even need to be a subsystem of $\mathcal{F}_{\left(P, e_{P}\right)}(G, b)$. We introduce a generalised category to circumvent this (see [9, Section 3] for proofs and details).

Definition 2.16. Let $G$ be a finite group, $N \unlhd G$ and $c$ a $G$-stable block of $k N$. A $(c, G)$-Brauer pair is a pair $\left(Q, e_{Q}\right)$, where $Q$ is a $p$-subgroup of $G$ with $\operatorname{Br}_{Q}^{N}(c) \neq 0$. Let $\left(P, e_{P}\right)$ be a maximal $(c, G)$-Brauer pair. For $Q \leq P$, let $e_{Q}$ 
be the unique block of $k C_{N}(Q)$ such that $\left(Q, e_{Q}\right) \leq\left(P, e_{P}\right)$. Denote the category on $P$ with morphisms consisting of all injective group homomorphisms $\varphi: Q \rightarrow R$ for which there is some $g \in G$ such that $\varphi(x)={ }^{g} x$ for all $x \in Q$ and ${ }^{g}\left(Q, e_{Q}\right) \leq\left(R, e_{R}\right)$ by $\mathcal{F}_{\left(P, e_{P}\right)}(G, N, c)$.

We refer to this category as the generalised Brauer category. If $G=N$ as in the previous definition, one obtains the usual fusion systems of blocks from Theorem 2.15. See [9, Theorem 3.4] for a proof that the generalised Brauer category defines a fusion system.

Theorem 2.17 ([9, Theorem 3.9]). Let $G$ be a finite group, $N \unlhd G$ and $c$ a $G$ stable block of $k N$. Let $\left(S, e_{S}^{\prime}\right)$ be a maximal $(c, G)$-Brauer pair. If b is a block of $k G$ covering $c$ with maximal $b$-Brauer pair $\left(P, e_{P}\right)$, we have the relations

$$
\begin{array}{rlrl}
P & \leq S, & \mathcal{F}_{\left(P, e_{P}\right)}(G, b) & \leq \mathcal{F}_{\left(S, e_{S}^{\prime}\right)}(G, N, c), \\
S \cap N & =P \cap N, & \mathcal{F}_{\left(S \cap N, e_{S \cap N}^{\prime}\right)}(N, c) \dot{\unlhd} \mathcal{F}_{\left(S, e_{S}^{\prime}\right)}(G, N, c) .
\end{array}
$$

It should be noted, that we believe that the generalised Brauer category is very likely to appear in prospective stronger reduction theorems.

Lemma 2.18 ([9, Lemma 6.1]). Let $G$ be a finite group with $N \unlhd G$ and $b$ a block of $k G$ with defect group $D$. Then there exists a block $c$ of $k N$, which is covered by $b$, having $D \cap N$ as a defect group.

We finish this section by making a link between fusion systems and blocks in stating the two known reduction theorems with respect to Conjecture 1.1.

Theorem 2.19 ([6, Theorem 3.1]). Let $\mathcal{F}$ be a reduction simple fusion system on a p-group $P$. Assume that $\operatorname{Aut}(P)$ is a p-group. If $G$ is a finite group having an $\mathcal{F}$-block, then there exists a quasisimple group $L$ with $p^{\prime}$-centre also having an $\mathcal{F}$-block.

Theorem 2.20 ([9, Theorem 4.2]). Let $\mathcal{F}_{1}$ and $\mathscr{F}_{2}$ be fusion systems on a $p$-group $P$ such that $\mathscr{F}_{1} \subseteq \mathcal{F}_{2}$. Assume that

(a) $\mathscr{F}_{1}$ is reduction simple,

(b) if $\mathcal{F}_{F}$ is a fusion system on $P$ containing $\mathcal{F}_{1}$, then $\mathcal{F}_{F}=\mathscr{F}_{1}$ or $\mathcal{F}=\mathscr{F}_{2}$,

(c) if $\mathcal{F}_{1}$ is a non-trivial normal subsystem of $\mathcal{F}_{2}$, then $\mathcal{F}=\mathcal{F}_{1}$ or $\mathcal{F}=\mathscr{F}_{2}$.

If there exists a finite group with an $\mathcal{F}_{1}$ or $\widetilde{F}_{2}$-block, then there also exists a quasisimple group $L$ with $p^{\prime}$-centre with an $\mathcal{F}_{1}$ or $\mathcal{F}_{2}$-block.

Note that neither of these reductions can be applied to the exotic Parker-Semeraro system $\mathcal{F}_{7}^{1}(6)$ on a Sylow 7 -subgroup $S$ of $G_{2}(7)$ (see [12, Notation 5.14]): 
Since its subsystems are in correspondence to the subgroups of $C_{6}$, it will not be possible to fit each subsystem into a pair fulfilling the assumptions of Theorem 2.20. Since $\operatorname{Aut}(S)$ is not a 7-group, Theorem 2.19 is also not applicable.

However, in the next section, we generalise Theorem 2.20 from two fusion systems to all subsystems of $p^{\prime}$-index of a certain fusion system.

\section{Reduction theorems}

We start this section by stating several reduction theorems which are essential for the study of fusion systems of blocks. These results are called respectively the first and second Fong reduction.

Proposition 3.1 ([1, Part IV, Proposition 6.3]). Let $\mathcal{F}$ be a fusion system on a $p$ group $P$, and let $G$ be a finite group having an $\mathcal{F}$-block $b$. Let $N \unlhd G$, and let $c$ be a block of $k N$ which is covered by $b$. Then the group $I(c)=\left\{g \in G \mid{ }^{g} c=c\right\}$ has an $\mathcal{F}$-block.

We use the first Fong reduction often in the following form.

Corollary 3.2. Let $\mathcal{F}$ be a fusion system, and let $G$ be a finite group possessing an $\mathcal{F}$-block $b$ such that $|G: Z(G)|$ is minimal among all finite groups having an $\mathscr{F}$-block. Then $b$ is inertial, i.e. it covers only $G$-stable blocks.

Proof. Choose $N, c$ as in Proposition 3.1. Since $Z(G) \subseteq I(c)$, this proposition implies directly that $b$ is inertial.

Theorem 3.3 ([1, Part IV, Theorem 6.6]). Let $G$ be a finite group with $N \unlhd G$ and $c$ a $G$-stable block of $N$ with trivial defect. Let $b$ be the block of $G$ covering $c$, and let $\left(P, e_{P}\right)$ be a maximal b-Brauer pair; then $N \cap P=1$, and there exists a central extension $1 \rightarrow Z \rightarrow \widetilde{G} \rightarrow G / N \rightarrow 1$, where $Z$ is a cyclic $p^{\prime}$-group. Furthermore, there is a block $\widetilde{b}$ of $k \widetilde{G}$ such that if we identify $P$ with the Sylow p-subgroup of the inverse image of $P N / N$ in $\widetilde{G}$, then there is a maximal $\widetilde{b}$-Brauer pair $\left(P, f_{P}\right)$ such that $\mathscr{F}_{\left(P, e_{P}\right)}(G, b)=\mathscr{F}_{\left(P, f_{P}\right)}(\widetilde{G}, \widetilde{b})$.

Lemma 3.4. Let $\mathcal{F}$ be a reduction simple fusion system and $G$ a finite group having an $\mathcal{F}$-block $b$ with non-abelian defect group P. If $G=\left\langle{ }^{g} P: g \in G\right\rangle$, then there exists a quasisimple group $L$ with $p^{\prime}$-centre having an $\mathcal{F}$-block.

Proof. We claim that if $N \unlhd G$ is proper, then $N$ has a block $d$ which is covered by $b$ and of defect zero. Indeed, by Lemma 2.18 , we can choose $d$ such that it has $P \cap N$ as defect group. Since $N$ is normal and each morphism in $\mathcal{F}$ is in- 
duced by conjugation with an element in $G, P \cap N$ is also strongly $\mathcal{F}$-closed. If $P \cap N \neq 1$, then, by reduction simplicity, $P \cap N=P$, which would imply that $N=G$, as $G=\left\langle{ }^{g} P \mid g \in G\right\rangle$. This contradiction implies $P \cap N=1$.

By Theorem 3.3, in this case, there is a $p^{\prime}$-central extension $\widetilde{G}$ of $G / N$ coming from an exact sequence $1 \rightarrow Z \rightarrow \widetilde{G} \rightarrow G / N \rightarrow 1$ having a block $c$ that is an $\mathscr{F}$-block. We now construct a quasisimple group $L$ with an $\mathscr{F}$-block. If we choose $N$ to be a maximal normal subgroup, then $G / N$ is either cyclic of prime order or $G / N$ is a non-abelian simple group. Note that, by simplicity of $G / N$, we necessarily have $Z=Z(\widetilde{G})$ in the extension above.

First, we assume that $G / N$ is cyclic; thus let $g \in G / N$ be the generating element and $\tilde{g}$ a preimage of $g$ in $\widetilde{G}$. Then $\widetilde{G}=\langle Z, \tilde{g}\rangle$. This means $\widetilde{G}$ is abelian, hence so is $P$, which is a contradiction.

So we are left with the case that $G / N$ is non-abelian simple. Define $L=[\widetilde{G}, \widetilde{G}]$. We have $L Z / Z \unlhd \widetilde{G} / Z=G / N$. First assume $L Z / Z=1$; then $L Z=Z$, which means that $L \subseteq Z$, which implies $\widetilde{G} / Z \subseteq \widetilde{G} / L$. This is a contradiction since $\widetilde{G} / L$ is abelian, and $\widetilde{G} / Z$, which is a homomorphic image of $G / N$, is not. So, by simplicity, $L Z / Z=\widetilde{G} / Z$, so $L Z=\widetilde{G}$. Taking commutators of this equation implies $[L, L]=[\widetilde{G}, \widetilde{G}]=L$, so $L$ is perfect. Since we have

$$
\widetilde{G} / Z=L Z / Z=L / L \cap Z,
$$

$L$ is also a $p^{\prime}$-central extension of $G / N$ by $Z \cap L$, and thus quasisimple.

We have $\widetilde{G}=L Z=L \times Z / K$ for some $p^{\prime}$-central subgroup $K$. This means there exists a bijection, preserving the associated fusion systems, between the blocks of $\widetilde{G}$ and the blocks of $L \times Z$ having $K$ in their kernel. But we can identify each block of $L \times Z$ with a block of $L$ since the blocks of $Z$ are just linear characters. It is easy to see that blocks which differ by tensoring with a linear character give rise to the same fusion system. In particular, there is a block of $L$ which is an $\widetilde{F}$-block.

Proof of Theorem 1.6. Assume $G$ to be of minimal order among the groups possessing an $\widetilde{F}_{H}$-block $b$ for some $H \leq \Gamma_{p^{\prime}}(\mathcal{F})$. Let $N \unlhd G$ and $c$ a block of $N$ covered by $b$. By Proposition 3.1 and our assumption, we may assume $G=I(c)$. In particular, we may assume that $c$ is $G$-stable and the unique block of $N$ covered by $b$.

Now $P$ is a $(b, G)$-defect group. Consider $M:=\left\langle{ }^{g} P \mid g \in G\right\rangle \unlhd G$. Let $d$ be the block of $k M$ covered by $b$. Since $d$ is $G$-stable, we have a homomorphism $G \rightarrow \operatorname{Aut}(k M d), g \mapsto c_{g}$, inducing a map $G \rightarrow \operatorname{Out}(k M d)$. Let $K$ be the kernel of this map. Clearly, $M \subseteq K$. We claim $K=G$.

Indeed, let $f$ be the block of $k K$ covered by $b$. Let $\left(P, e_{P}\right)$ be a maximal $b$-Brauer pair and $\left(S, e_{S}^{\prime}\right)$ a maximal $(G, K, f)$-Brauer pair such that $P \leq S$. 
By [11, Section 5], $G / K$ is a $p^{\prime}$-group. Thus, we may assume $P=S$. Furthermore, $\widetilde{F}_{H} \leq \mathcal{F}_{\left(P, e_{P}^{\prime}\right)}(G, K, f)$ and $\widetilde{F}_{\left(P, e_{P}^{\prime}\right)}(K, f) \dot{\unlhd} \mathcal{F}_{\left(P, e_{P}^{\prime}\right)}(G, K, f)$ by Theorem 2.17 .

Thus, by assumption (b), $\mathcal{F}_{\left(P, e_{P}^{\prime}\right)}(G, K, f)$ is of $p^{\prime}$-index in $\mathcal{F}_{\text {r too, and hence }}$ there is some $H^{\prime} \leq \Gamma_{p^{\prime}}(\mathcal{F})$ such that $\mathcal{F}_{\left(P, e_{P}^{\prime}\right)}(G, K, f)=\mathscr{F}_{H^{\prime}}$. Similarly, by assumption (c), there is also a $J \leq \Gamma_{p^{\prime}}(\mathscr{F})$ such that $\mathscr{F}_{\left(P, e_{P}^{\prime}\right)}(K, f)=\mathcal{F}_{J}$. By the minimality of $G$, we deduce $G=K$.

By this observation, $G$ acts as inner automorphisms on $k M d$. Thus, by [10, Theorem 7], $k M d$ and $k G b$ have isomorphic source algebras. By [6, Proposition 2.12], we have that $d$ is an $\widetilde{F}_{H}$-block as well. Using the minimality once more, we obtain $G=M$. The previous lemma implies the theorem since reduction simplicity of $\mathcal{O}^{p^{\prime}}(\mathcal{F})$ implies reduction simplicity of $\mathscr{F}$ for a fusion system $\mathcal{F}$.

Note that we obtain [9, Theorem 4.2] as a corollary of the theorem we just proved by setting $\Gamma_{p^{\prime}}(\mathcal{F})=C_{2}$ respectively $\Gamma_{p^{\prime}}(\mathcal{F})=1$.

We finish this section with proving Theorem 1.5, which further restricts the structure of reduction simple fusion systems.

Proof of Theorem 1.5. Let a group $\widetilde{G}$ have an $\widetilde{F}$-block $\widetilde{b}$ subject to $|\widetilde{G}: Z(\widetilde{G})|$ being minimal, and let $M \unlhd \widetilde{G}$ be maximal such that $P \nsubseteq M$. Note that, since each normal $p$-subgroup is contained in each defect group of a $p$-block, we have that $O_{p}(\widetilde{G}) \leq P$. Furthermore, $O_{p}(\widetilde{G})$ is strongly $\widetilde{F}$-closed, so either $O_{p}(\widetilde{G})=1$ or $O_{p}(\widetilde{G})=P$. If $O_{p}(\widetilde{G})=P$, then $Z(P) \unlhd \widetilde{G}$. In particular, $Z(P)$ is strongly $\mathcal{F}$-closed, which is not possible since $P$ is non-abelian and $\mathcal{F}$ is reduction simple. Thus, $O_{p}(\widetilde{G})=1$. In particular, $Z(\widetilde{G})$ is a $p^{\prime}$-group. By maximality, we must have $Z(\widetilde{G}) \subseteq M$. Note that $P \cap M=1$ by reduction simplicity, but $P \cap M$ is a defect group of a block of $M$, which is covered by $\widetilde{b}$ by Lemma 2.18. In particular, there is a central extension $1 \rightarrow Z \rightarrow G \stackrel{\pi}{\rightarrow} \widetilde{G} / M \rightarrow 1$ for some central $p^{\prime}$-group $Z$ such that $G$ has an $\mathcal{F}$-block $b$ by Theorem 3.3 (with the roles of $G$ and $\widetilde{G}$ interchanged).

Now we check that $G$ satisfies the claims (a), (b) and (c) from Theorem 1.5. To prove claim (a), note that $|G: Z(G)| \leq|G: Z|=|\widetilde{G}: M| \leq|\widetilde{G}: Z(\widetilde{G})|$. In particular, by Corollary 3.2, $b$ is inertial.

Suppose $H \unlhd G$ with $P \nsubseteq H$. We show $H \subseteq Z(G)$. We may assume $Z \subsetneq H$. Let $\varepsilon: \widetilde{G} \rightarrow \widetilde{G} / M$ be the canonical surjection. The maps $\varepsilon$ and $\pi$ induce bijections between the set of subgroups of $\widetilde{G}$ containing $M$ and the set of subgroups of $G$ containing $Z$, which preserves normality. This bijection sends $H$ to $\pi^{-1}(\varepsilon(H))$. Furthermore, $P \subseteq H$ if and only if $P \subseteq \pi^{-1}(\varepsilon(H))$. Since there is no normal subgroup of $\widetilde{G}$ properly containing $M$ and not containing $P$, it follows that there is no normal subgroup of $G$ properly containing $Z$ and not containing $P$, which proves claim (b). In particular, $Z(G)$ is a $p^{\prime}$-group. 
Note that we have $O_{p}(G)=1$ for any $G$ having an $\mathcal{F}$-block. Since

$$
F(G)=\prod_{q \in \mathbb{P}} \prod_{Q \in \operatorname{Syl}_{q}(F(G))} Q,
$$

we thus have $\operatorname{Syl}_{p}(F(G))=1$. Thus, by the above, $F(G) \subseteq Z(G)$, so in fact $F(G)=Z(G)$.

Now let $c$ be the block of $E(G)$ which is covered by $b$. If $E(G) \cap P=1$, $E(G) \subseteq Z(G)$ again by the above. But then $E(G)=1$ and $F(G)$ is central, which would mean that $G$ is abelian. So $E(G) \cap P \neq 1$ and thus $P \subseteq E(G)=L_{1} \cdots L_{t}$, where $\left\{L_{1}, \ldots, L_{t}\right\}$ are the components of $G$. We have

$$
\begin{aligned}
& E(G) \cong\left(L_{1} \times \cdots \times L_{t}\right) / K \\
& \quad \text { for } K \subseteq Z\left(L_{1} \times \cdots \times L_{t}\right)=Z\left(L_{1}\right) \times \cdots \times Z\left(L_{t}\right) .
\end{aligned}
$$

We claim that $K$ is a $p^{\prime}$-group. It suffices to prove $O_{p}\left(L_{i}\right)=1$ for each $1 \leq i \leq t$. Indeed, if we assume the contrary, then the group $O_{p}\left(L_{1}\right) \cdots O_{p}\left(L_{t}\right)$ is a nontrivial normal subgroup of $E(G)$. In particular, $O_{p}(E(G)) \neq 1$. However, this is a characteristic subgroup of the layer, which implies $O_{p}(G) \neq 1$, a contradiction.

Thus, there is a fusion system preserving bijection between the blocks of $E(G)$ and the blocks of $L_{1} \times \cdots \times L_{t}$ which have $K$ in their kernel. In particular, we may assume $P=P_{1} \times \cdots \times P_{t}$ and $c=c_{1} \times \cdots \times c_{t}$, where, for $1 \leq i \leq t, P_{i}$ is a defect group of the block $c_{i}$, which is a block of $L_{i}$ covered by $c$. If $r$ is the rank of $Z(P)$, then at most $r$ of the blocks $c_{i}$ can have non-trivial defect. Let $s \leq r$ be such that $s$ of the blocks $c_{i}$ have non-trivial defect. After possibly reordering, we may assume these are $c_{1}, \ldots, c_{s}$. We claim $L_{s+1} \cdots L_{t} \unlhd G$. Indeed, the conjugation action of $G$ on its components induces a group homomorphism $\sigma: G \rightarrow \operatorname{Sym}\left(\left\{L_{1}, \ldots, L_{t}\right\}\right) \cong \mathbb{S}_{t}$ as follows: $\sigma(x)(i):=j$ if and only if ${ }^{x} L_{i}=L_{j}$. Assume there is an $x \in G$ such that ${ }^{x} L_{i}=L_{j}$ for $i \leq s, j>s$. Since $b$ is inertial, $c$ is $G$-stable. This means ${ }^{x} c=c$, so

$$
{ }^{x} c_{1} \times \cdots \times{ }^{x} c_{t}=c_{1} \times \cdots \times c_{t},
$$

but this implies ${ }^{x} P_{i}$ is non-trivial. This contradiction implies normality. Now we can apply the above to deduce $L_{s+1} \cdots L_{t}=1$, which implies claim (c).

We can further restrict the structure of reduction simple fusion systems by specialising to the case of $Z(P)$ being cyclic.

Theorem 3.5. Let $P$ be a non-abelian p-group such that $Z(P)$ is cyclic, and let $\mathcal{F}$ be a reduction simple fusion system on $P$. If $\mathcal{F}$ is block-realisable, then there exists a fusion system $\mathcal{F}_{0}$ on $P$ and a quasisimple group $L$ with an $\mathcal{F}_{0}$-block, where $\mathcal{O}_{p}\left(\mathcal{F}_{0}\right)=1$. 
Proof. Let $G$ be a finite group having an $\mathcal{F}$-block $b$ with defect group $P$. We may choose $G$ such that the conclusions of Theorem 1.5 hold. Let $L=\left\langle{ }^{g} P \mid g \in G\right\rangle$. Thus, since $P \subseteq E(G)$ as in the proof of Theorem 1.5, we have $L \unlhd E(G)$. By Theorem 1.5, the number of components of $G$ is bounded by the rank of $Z(P)$. By cyclicity of that group, $E(G)$ is quasisimple. Furthermore, $L$ is non-central, so we must have $L=E(G)$ is quasisimple.

Let $d$ be the block of $k L$ which is covered by $b$. Define $K$ to be the kernel of the map $G \rightarrow \operatorname{Out}(k L d)$, which is induced by $G \rightarrow \operatorname{Aut}(k L d), g \mapsto c_{g}$, and assume $K$ has a block $c$ which is covered by $b$. We get the triangle relations $\mathcal{F} \subseteq \mathcal{F}_{\left(P, e_{P}^{\prime}\right)}(G, K, c)$ and $\mathcal{F}_{\left(P, e_{P}^{\prime}\right)}(K, c) \dot{\unlhd} \mathcal{F}_{\left(P, e_{P}^{\prime}\right)}(G, K, c)=: \widetilde{\mathcal{F}}_{\text {as }}$ in the proof of Theorem 1.6. In the same fashion as in this theorem, application of [11, Theorem 7] and [6, Proposition 2.12] also implies

$$
\mathcal{F}_{\left(P, e_{P}^{\prime}\right)}(K, c) \cong \mathcal{F}_{\left(P, f_{P}\right)}(L, d)=: \mathcal{F}_{0} .
$$

We have $\mathcal{O}_{p}(\mathcal{F})=1$ by reduction simplicity. So Lemma $2.8 \operatorname{implies} \mathcal{O}_{p}(\widetilde{\mathcal{F}})=1$ and Lemma 2.9 implies $\mathcal{O}_{p}\left(\mathcal{F}_{0}\right)=1$.

\section{The Parker-Semeraro exotic fusion systems}

In this section, we use what we have developed so far to prove block-exoticity of the exotic Parker-Semeraro fusion systems.

In this chapter, $S$ will denote a Sylow 7-subgroup of $G_{2}(7)$. As in the introduction, by a Parker-Semeraro system, we mean a fusion system $\mathcal{F}$ on $S$ such that $\mathcal{O}_{p}(\mathcal{F})=1$. In what follows, we will use the notation of [12], where these systems have been classified.

We start by proving that it is not possible for most finite quasisimple groups to have a block with $S \in \operatorname{Syl}_{7}\left(G_{2}(7)\right)$ as a defect group. Fix $M$ to be the Monster group for the rest of this section.

Proposition 4.1. Let $S \in \mathrm{Syl}_{7}\left(G_{2}(7)\right)$. Assume $G$ is a finite quasisimple group having a block with defect group $S$. Then either $G=M$ or $G=G_{2}(7)$.

Most work will have to be done to deal with groups of Lie type. We are going to restate a lemma from [9], which will be very useful to deal with these groups.

Lemma 4.2 ([9, Lemma 6.2]). Let $H=L D$ be a finite group such that $L \unlhd H$ and $D$ is a p-group. Furthermore, let $c$ be a $D$-stable block of $k L$ with defect group $D \cap L$ and $\operatorname{Br}_{D}^{H}(c) \neq 0$, and let $D^{\prime}$ be such that $D \cap L \leq D^{\prime} \leq D$. Then

(a) c is a block of $L D^{\prime}$ with defect group $D^{\prime}$,

(b) if $D^{\prime}$ acts on $L$ as elements of $\operatorname{Inn}(L)$, then $D^{\prime}=\left(D^{\prime} \cap L\right) C_{D^{\prime}}(L)$. 
Proposition 4.3. Let $G$ be a quasisimple finite group, and denote the quotient $G / Z(G)$ by $\bar{G}$. Suppose $\bar{G}=G(q)$ is a finite group of Lie type, and let $p$ be a prime number $\geq 7,(p, q)=1$. Let $D$ be a p-group such that $Z(D)$ is cyclic of order $p$ and $Z(D) \subseteq[D, D]$. If $D$ is a defect group of a block of $G$, then there are $n, k \in \mathbb{N}$ and a finite group $H$ with

$$
\mathrm{SL}_{n}\left(q^{k}\right) \leq H \leq \mathrm{GL}_{n}\left(q^{k}\right) \quad\left(\text { or } \mathrm{SU}_{n}\left(q^{k}\right) \leq H \leq \operatorname{GU}_{n}\left(q^{k}\right)\right)
$$

such that there is a block $c$ of $H$ with non-abelian defect group $D^{\prime}$ such that $D^{\prime} / Z$ is of order $|D / Z(D)|$ for some $Z \leq D^{\prime} \cap Z(H)$.

Proof. Suppose $G$ has a block with defect group $D$. Then the Sylow $p$-subgroups of $\bar{G}$ cannot be abelian, which implies that the Weyl group of the algebraic group corresponding to $\bar{G}$ has an order divisible by $p$; see [5, Theorem 4.10.2(a)]. This implies that the exceptional part of the Schur multiplier of $\bar{G}$ is trivial; see [5, Table 6.1.3]. Thus, there is a simple simply connected algebraic group $\bar{K}$ defined over $\overline{\mathbb{F}_{q}}$ and a Frobenius morphism $F: \bar{K} \rightarrow \bar{K}$ such that $\bar{K} F$ is a central extension of $G$. If $\bar{K}$ is of type $A$, set $H:=\bar{K} F$ and $c$ to be the block whose image has defect group $D$ under the algebra homomorphism $k H \rightarrow k G$ induced by $H \rightarrow G$.

Thus, assume $\bar{K}$ is not of type $A$. Since the kernel of $K^{F} \rightarrow \bar{K}^{F}$ is a $p^{\prime}$-group, we have $\left|\bar{K}^{F}\right|_{p}=\left|K^{F}\right|_{p}$, so we may assume $G=\bar{K}^{F}$.

Denote a generator of $Z(D)$ by $z$. By Brauer's first main theorem, see [7, Theorem 3.6], we may assume the group $C_{G}(z)$ has a block $b$ with defect group $D$. Since $\bar{K}$ is simply connected, $C_{\bar{K}}(z)$ is a Levi subgroup of $\bar{K}$. If we denote $\bar{Z}:=Z\left(C_{\bar{K}}(z)\right)^{\circ}$, it is a well-known fact that $C_{\bar{K}}(z)=\left[C_{\bar{K}}(z), C_{\bar{K}}(z)\right] \bar{Z}$. The latter commutator is simply connected and thus a direct product of its components, which are simply connected as well and permuted by $F$. In particular, we have a decomposition $\left[C_{\bar{K}}(z), C_{\bar{K}}(z)\right]=\prod_{i=1}^{t} \prod_{j=1}^{r_{i}} \bar{L}_{i j}$, where each $\bar{L}_{i j}$ is simply connected simple, and the set of these groups for a fixed $i$ lie in the same $F$-orbit.

Set $L_{i}=\left(\prod_{j=1}^{r_{i}} \bar{L}_{i j}\right)^{F}$. Then we have $C_{G}(z)=\left(L_{1} \times \cdots \times L_{t}\right) T^{F}$, for an $F$-stable maximal torus $T \leq C_{\bar{K}}(z)$.

Now $T^{F}$ is abelian, and we have

$$
D / D \cap\left(L_{1} \times \cdots \times L_{t}\right) \cong T^{F} / T^{F} \cap\left(L_{1} \times \cdots \times L_{t}\right) .
$$

So $[D, D] \leq D \cap\left(L_{1} \times \cdots \times L_{t}\right) \neq 1$. By Lemma 2.18 , the latter group is furthermore a defect group of a block of $L_{1} \times \cdots \times L_{t}$. Now defect groups respect direct products and $Z(D) \cong C_{p}$. Thus, we may assume $D \cap\left(L_{1} \times \cdots \times L_{t}\right) \leq L_{1}$. In particular, $[D, D] \leq L_{1}$, and we may assume $Z(D) \leq L_{1}$. Since $Z(D)$ is central in $C_{G}(z)$, each $\bar{L}_{1 j}$ is of type $A$ and Lie rank at least $p$, so $L_{1}$ is isomorphic to either $\mathrm{SL}_{n}\left(q^{k}\right)$ or $\mathrm{SU}_{n}\left(q^{k}\right)$. 
Let $x \in D \backslash Z(D)$. We want to show that $x$ does not centralise $L_{1}$. First note if $\bar{L}=\mathrm{SL}_{n}\left(\overline{\mathbb{F}_{q}}\right)$ and $\sigma$ a Frobenius morphism, then $\bar{L}^{\sigma}$ is either $\operatorname{SL}_{n}\left(q^{k}\right)$ or $\operatorname{SU}_{n}\left(q^{k}\right)$ for some $k$, and we have $C_{\bar{L}}\left(\bar{L}^{\sigma}\right) \leq Z(\bar{L})$. Using the decomposition from above, we may write $x=\left(\prod_{i=1}^{t} \prod_{j=1}^{r_{i}} x_{i j}\right) t_{x}$ for $x_{i j} \in \bar{L}_{i j}, t_{x} \in Z(\bar{L})$. Let $y \in D$ such that $[x, y] \neq 1$, and write $y=\left(\prod_{i=1}^{t} \prod_{j=1}^{r_{i}} y_{i j}\right) t_{y}$ for $y_{i j} \in \bar{L}_{i j}, t_{y} \in Z(\bar{L})$. We have $[D, D] \leq L_{1}$, which means $\left[x_{11}, y_{11}\right] \neq 1$, so $x_{11}$ does not centralise $\bar{L}_{11}$ as well as $\bar{L}_{11}^{F^{r_{1}}}$ by the above and thus also not $L_{1}$.

Now let $c$ be a block of $k L_{1}$ covered by $b$; then we may assume that $c$ is $D$ stable, $\operatorname{Br}_{D}^{L_{1} D}(c) \neq 0$ and $D \cap L_{1}$ is a defect group of $c$; see Lemma 2.18. Let $D_{0}$ be the kernel of the map $D \rightarrow \operatorname{Out}\left(L_{1}\right)$. Then $Z(D) \leq\left(D \cap L_{1}\right) \leq D_{0}$. Now if we apply Lemma 4.2 , we obtain $D_{0}=\left(D_{0} \cap L_{1}\right) C_{D_{0}}\left(L_{1}\right)$. But we have seen that $C_{D}\left(L_{1}\right)=Z(D) \leq L_{1}$. So $D_{0} \leq L_{1}$.

If $D_{0}=D$, we can take $H=L_{1}, D^{\prime}=D$ and $Z=1$, and the claim holds. Thus, assume $D \neq D_{0}$. The elements of $T^{F}$ induce diagonal automorphisms of $L_{1}$. For special linear or unitary groups, the group of diagonal automorphisms modulo inner automorphisms is cyclic. In particular, we have that $D / D_{0}$ is cyclic. Let $D / D_{0}=\langle\bar{y}\rangle$. Let $\eta \in \mathrm{GL}_{n}\left(q^{k}\right)_{p}$ (respectively $\left.\mathrm{GU}_{n}\left(q^{k}\right)_{p}\right)$ such that ${ }^{\eta} u={ }^{y} u$ for $u \in L_{1}$. In particular, $\eta$ stabilises $c$. Define $H:=L_{1}\langle\eta\rangle$ to obtain $H$ as in the claim. Furthermore, define $D^{\prime}:=\left\langle D_{0}, \eta\right\rangle \leq H$. We also have $C_{L_{1}}(D)=C_{L_{1}}\left(D^{\prime}\right)$, so $\operatorname{Br}_{D^{\prime}}^{H}(c) \neq 0$. Now $H=L_{1} D^{\prime}$ and $D_{0}$ (@D $\cap L_{1}$ by construction) is a defect group of $c$ as a block of $k L_{1}$. Thus, we can apply Lemma 4.2 to obtain that $c$ is a block of $k H$ with defect group $D^{\prime}$.

We have $D^{\prime}=\left\langle D_{0}, \eta\right\rangle$ and $D=\left\langle D_{0}, y\right\rangle$. The canonical maps $D^{\prime} \rightarrow \operatorname{Aut}\left(L_{1}\right)$ and $D \rightarrow \operatorname{Aut}\left(L_{1}\right)$ have the same image. Thus,

$$
D^{\prime} / C_{D^{\prime}}\left(L_{1}\right) \cong D / C_{D}\left(L_{1}\right)=D / Z(D) .
$$

Define $Z:=Z\left(\operatorname{GL}_{n}\left(q^{k}\right)\right) \cap D^{\prime}$ or $Z:=Z\left(\operatorname{GU}_{n}\left(q^{k}\right)\right) \cap D^{\prime}$, so $Z=C_{D^{\prime}}\left(L_{1}\right)$, which gives $D^{\prime} / Z \cong D / Z(D)$. In particular, $D^{\prime}$ is non-abelian since $y$ acts nontrivially on $D_{0}$.

Proposition 4.4. If $G$ is as in the previous proposition, then $G$ has no blocks with defect groups isomorphic to a Sylow 7-subgroup of $G_{2}(7)$.

Proof. Assume $D \in \operatorname{Syl}_{7}\left(G_{2}(7)\right)$, in particular $|D|=7^{6}$ and $Z(D) \cong C_{7}$. Let $H, D^{\prime}$ be as in the assertion of the previous proposition with $p=7$. Assume first $H \leq \operatorname{GL}_{n}\left(q^{k}\right)$, and let $a$ be such that $\left|q^{k}-1\right|_{7}=7^{a}$. Then, since $\mathrm{SL}_{n}\left(q^{k}\right) \leq H$, we have

$$
\begin{aligned}
\left|D^{\prime}\right| & =|D / Z(D)| \cdot|Z| \\
& =7^{5}|Z| \leq 7^{5}|Z(H)| \leq 7^{5}\left|Z\left(\operatorname{SL}_{n}\left(q^{k}\right)\right)\right| \leq 7^{5+a} .
\end{aligned}
$$


Now the block of $k \mathrm{GL}_{n}\left(q^{k}\right)$ covering $c$ has a defect group of order at most $7^{2 a+5}$. But it is a well-known fact that (non-abelian) defect groups of $\mathrm{GL}_{n}\left(q^{k}\right)$ have order at least $7^{7 a+1}$; see [4, Theorem $3 \mathrm{C}$ ]. Thus, $7^{7 a+1} \leq 7^{2 a+5}$, which is a contradiction. The case $H \leq \mathrm{GU}_{n}\left(q^{k}\right)$ can be shown in the same fashion by considering the 7-part of $q^{k}+1$ instead of $q^{k}-1$.

We use this observation to prove Proposition 4.1.

Proof of Proposition 4.1. By the previous proposition, we may assume $G$ is not a group of Lie type in characteristic not equal to 7 .

First, assume $G / Z(G)$ is an alternating group $\mathfrak{A}_{m}$. Then $S$ is isomorphic to a Sylow 7-subgroup of some symmetric group $\Im_{7 w}$ with $w \leq 6$. Define the cycle $\sigma_{i}=((i-1) 7+1, \ldots, i 7)$ and the subgroup $S^{\prime}=\left\langle\sigma_{1}, \ldots, \sigma_{6}\right\rangle \leq \mathfrak{A}_{m}$. Then $S^{\prime} \in \operatorname{Syl}_{7}\left(\mathfrak{U}_{m}\right)$. But this group is abelian, which means that $S \notin \operatorname{Syl}_{7}\left(\mathfrak{A}_{m}\right)$.

Next, assume $G$ is a group of Lie type over a field of characteristic $p=7$. In this case, $Z(G)$ is a $7^{\prime}$-group, and we may assume that $G=\mathbf{G}^{F}$, where $\mathbf{G}$ is simple and simply connected, $F$ is a Frobenius morphism. Furthermore, $S \in \operatorname{Syl}_{7}(G)$ by [2, Theorem 6.18$]$. We first deal with the classical groups. First consider type $B_{n}$; here we have $|G|_{p}=q^{n^{2}}$, which can be equal to $7^{6}$ only if $n=1$, which is not possible since $n>1$ for these groups. The case is the same for the groups of type $C_{n}$. For type $D_{n}$ and ${ }^{2} D_{n}$, we have $|G|_{p}=q^{n(n-1)}$. Since $n>3, p^{6}$ is also no possibility here. Finally, consider types $A_{n}$ and ${ }^{2} A_{n}$; here we have $|G|_{p}=q^{\frac{1}{2}(n+1) n}$. If $n=1$, these groups have abelian Sylow $p$-subgroups, and if $n \geq 4$, the order is too big. Thus, the only possibilities are $n=2$ or $n=3$. However, in these cases, we obtain Sylow 7-subgroups which are conjugate to the groups of upper unitary triangle matrices. Hence, these groups have nilpotency class 2 respectively 3 . However, the nilpotency class of $S$ is 5 . This leaves us with the exceptional groups of Lie type. Looking at their orders, we can directly exclude the exceptional Steinberg groups, the Suzuki, Ree and Tits groups. For the exceptional Chevalley groups, $G_{2}(7)$ is the only possibility.

Finally, if $G / Z(G)$ is sporadic, the monster $M$ is the only group with a 7-part big enough to contain $S$, which implies our claim.

This result can be used to achieve a reduction specifically for the Parker-Semeraro systems.

Lemma 4.5. Let $\mathcal{F}$ be an exotic Parker-Semeraro system. Then $\mathcal{F}$ is reduction simple.

Proof. Assume $1 \neq N \leq S$ is strongly $\mathscr{F}$-closed. In particular, $N \unlhd S$, which implies $Z(S) \leq N$. Thus, as in the proof of [12, Theorem 6.2], $N=S$. 
We will use the reduction simplicity of the Parker-Semeraro systems in the following theorem.

Theorem 4.6. Suppose there is an exotic Parker-Semeraro system $\mathcal{F}$ which is block-realisable. Then there is an exotic Parker-Semeraro system $\mathcal{F}_{0}$ which is block-realisable by a block of a quasisimple group.

Proof. Assume $G$ is a finite group having an $\mathcal{F}$-block $b$. We may choose $G$ such that the conclusions of Theorem 1.5 hold, with $S \in \operatorname{Syl}_{7}\left(G_{2}(7)\right)$ in the role of $P$. Define $L=\left\langle{ }^{g} S \mid g \in G\right\rangle \unlhd G$. Since $Z(S)$ is cyclic of order $p, \mathcal{F}$ satisfies the hypothesis of Theorem 3.5 with $P=S$. Arguing as in the proof of that theorem, $L=E(G)$ is quasisimple, and there is a block of $k L$ with defect group $S$ and fusion system $\mathcal{F}_{0}$ such that $O_{p}\left(\mathcal{F}_{0}\right)=1$. So $\mathscr{F}_{0}$ is a Parker-Semeraro system.

If $\mathcal{F}_{0}$ is exotic, we are done. Suppose $\mathcal{F}_{0}$ is realisable. So either $\mathcal{F}_{0}=\mathcal{F}_{S}(M)$ or $\mathcal{F}_{0}=\mathscr{F}_{S}\left(G_{2}(7)\right)$. By Proposition $4.1, L=M$ or $L=G_{2}(7)$, and hence $L$ is simple.

We claim $G=L$. Consider the map $\varphi: G \rightarrow \operatorname{Out}\left(F^{*}(G)\right)$, and let $g \in \operatorname{ker}(\varphi)$. Then there exists $x \in F^{*}(G)$ such that $y \in F^{*}(G)$ with $g y g^{-1}=x y x^{-1}$, i.e.

$$
x^{-1} g \in C_{G}\left(F^{*}(G)\right)=Z\left(F^{*}(G)\right) \text {. }
$$

This implies $\operatorname{ker} \varphi=F^{*}(G)$ and thus $G / F^{*}(G) \cong \operatorname{Out}_{G}\left(F^{*}(G)\right)$. But in our case, we have $F^{*}(G)=Z(G) E(G)$, so $\operatorname{Out}_{G}\left(F^{*}(G)\right)=\operatorname{Out}_{G}(E(G))$. However, this group needs to be trivial since $\operatorname{Out}(M)=\operatorname{Out}\left(G_{2}(7)\right)=1$. This implies $G=Z(G) E(G)$ and, in either case, $G=Z(G) \times L$. By choice of $G$, the claim follows.

We now claim that, in either of these cases, $b$ needs to be the principal block. For $G_{2}(7)$, this is a well known fact; see e.g. [8, Example 3.8]. So assume $G=M$. We want to compute $C_{N_{M}(P)}(S)$, where $P$ is a subgroup of order 7 of the monster. We know that $C_{N_{M}(P)}(S)=Z(S) \times O_{7^{\prime}}\left(C_{N_{M}(P)}(S)\right)$. Furthermore, by [13, Theorem 1.1], $N_{M}(P)$ is 7-constrained, i.e. $C_{N_{M}(P)}\left(O_{7}\left(N_{M}(P)\right)\right) \subseteq O_{7}\left(N_{M}(P)\right)$. Also by [13, Theorem 1.1], $N_{M}(P)=7^{1+4}\left(2 \Im_{7} \times 3\right)$, i.e. $O_{7}\left(N_{M}(P)\right)=7^{1+4}$ and thus $C_{N_{M}(P)}(S) \cong C_{p}$. But this means $C_{M}(S) / Z(S)$ is trivial, and since the $N_{M}(S)$-classes of irreducible characters of this group are in 1:1-correspondence with blocks of $M$ with defect group $S$, the claim follows. However, this implies that $\mathcal{F}$ cannot be exotic, which is a contradiction.

We use this to deduce block-exoticity of the Parker-Semeraro systems.

Proof of Theorem 1.2. Let $S$ be a Sylow 7-subgroup of $G_{2}(7)$, and let $\mathcal{F}$ be one of the exotic Parker-Semeraro systems. Assume $G$ is a group with an $\mathscr{F}$-block. 
By the previous theorem, we may assume that $G$ is quasisimple. Let $A$ be the simple quotient of $G$. By Proposition 4.1, we may assume either $A=G_{2}(7)$ or $A=M$, and thus, since $\operatorname{Out}(A)=1$ in both these cases, also either $G=G_{2}(7)$ or $G=M$. But as in the proof of the previous theorem, this means that the $\mathcal{F}$-block is principal, which is not possible for an exotic fusion system.

\section{Bibliography}

[1] M. Aschbacher, R. Kessar and B. Oliver, Fusion Systems in Algebra and Topology, London Math. Soc. Lecture Note Ser. 391, Cambridge University, Cambridge, 2011.

[2] M. Cabanes and M. Enguehard, Representation Theory of Finite Reductive Groups, New Math. Monogr. 1, Cambridge University, Cambridge, 2004.

[3] D. A. Craven, The Theory of Fusion Systems. An Algebraic Approach, Cambridge Stud. Adv. Math. 131, Cambridge University, Cambridge, 2011.

[4] P. Fong and B. Srinivasan, The blocks of finite general linear and unitary groups, Invent. Math. 69 (1982), no. 1, 109-153.

[5] D. Gorenstein, R. Lyons and R. Solomon, The Classification of the Finite Simple Groups, Math. Surveys Monogr. 40, American Mathematical Society, Providence, 1994.

[6] R. Kessar, The Solomon system $\mathscr{F}_{\text {Sol }}(3)$ does not occur as fusion system of a 2-block, J. Algebra 296 (2006), no. 2, 409-425.

[7] R. Kessar, Introducton to block theory, in: Group Representation Theory, EPFL Press, Lausanne (2007), 47-77.

[8] R. Kessar and G. Malle, Local-global conjectures and blocks of simple groups, in: Groups St Andrews 2017 in Birmingham, London Math. Soc. Lecture Note Ser. 455, Cambridge University, Cambridge (2019), 70-105.

[9] R. Kessar and R. Stancu, A reduction theorem for fusion systems of blocks, J. Algebra 319 (2008), no. 2, 806-823.

[10] B. Külshammer, Morita equivalent blocks in Clifford theory of finite groups, Astérisque (1990), no. 181-182, 209-215.

[11] B. Külshammer, Donovan's conjecture, crossed products and algebraic group actions, Israel J. Math. 92 (1995), no. 1-3, 295-306.

[12] C. Parker and J. Semeraro, Fusion systems over a Sylow $p$-subgroup of $\mathrm{G}_{2}(p)$, Math. Z. 289 (2018), no. 1-2, 629-662.

[13] C. W. Parker and C. B. Wiedorn, A 7-local identification of the Monster, Nagoya Math. J. 178 (2005), 129-149. 
Received February 21, 2019; revised October 6, 2019.

\section{Author information}

Patrick Serwene, Department of Mathematics, City, University of London, Northampton Square, London, EC1V 0HB, United Kingdom.

E-mail: patrick.serwene@city.ac.uk 